\title{
Féeries
}

Études sur le conte merveilleux, XVII $-\mathrm{XIX}{ }^{\mathrm{e}}$ siècle

14 | 2017

Conte merveilleux et poésie

\section{Introduction. - Poète, même en prose, même en vers}

Bertrand Vibert

\section{(2) OpenEdition}

1 Journals

Édition électronique

URL : http://journals.openedition.org/feeries/1056

DOI : $10.4000 /$ feeries. 1056

ISSN : 1957-7753

Éditeur

UGA Éditions/Université Grenoble Alpes

Édition imprimée

ISBN : 978-2-37747-012-9

ISSN : 1766-2842

Référence électronique

Bertrand Vibert, «Introduction. - Poète, même en prose, même en vers », Féeries [En ligne], 14 | 2017, mis en ligne le 31 juillet 2017, consulté le 23 septembre 2020. URL : http://journals.openedition.org/ feeries/1056 ; DOI : https://doi.org/10.4000/feeries.1056

Ce document a été généré automatiquement le 23 septembre 2020.

() Féeries 


\title{
Introduction. - Poète, même en prose, même en vers
}

\author{
Bertrand Vibert
}

\section{Lisons Andersen :}

Je trouve que le genre du conte est le royaume le plus étendu de la poésie. Il va des tombes des temps anciens, où le sang est encore chaud, au livre d'images des légendes enfantines et pieuses, il englobe la littérature populaire et celle qui est du ressort de l'art, il représente pour moi toute la poésie, et celui qui est capable de le pratiquer, doit pouvoir y mettre le tragique, le comique, le naïf, l'ironie et l'humour, et il a à son service la corde lyrique, le récit destiné aux enfants et la langue de celui qui décrit la nature ${ }^{1}$.

2 On ne saurait prononcer plus fervent acte de foi dans le pouvoir du conte nimbé de l'aura de la poésie, celle-ci étant considérée à la fois comme valeur et comme étalon des valeurs esthétiques. Faire de la poésie le principe de légitimation du conte et même le tout de la littérature, voilà qui signe une esthétique romantique, et qui ne déparerait pas non plus dans un credo symboliste. Mais une déclaration aussi accueillante et englobante ne simplifie guère a priori la tâche du critique ou du poéticien. Car au-delà de ce charme unifiant de la poésie, que faut-il mettre sous le nom même de "poésie »? et sous celui de "conte»? S'agissant d'Andersen, on peut se référer à sa propre pratique et tenter de la circonscrire. Mais ce n'est justement pas ce à quoi il nous invite, en revendiquant pour le genre des caractéristiques opposées qui se jouent des définitions. Déclinons donc quelques perspectives qui mettent à mal les frontières du conte :

- une transhistorique, qui relativise beaucoup la stabilité des traits que le «nom de genre» peut recouvrir puisque, ainsi que l'a montré Jean-Marie Schaeffer ${ }^{2}$, la permanence d'un nom de genre n'équivaut pas à une essence de genre ;

- une transgénérique, précisément, car on voit bien qu'Andersen fait toucher le conte aux mythes « des temps anciens » et aux « légendes » - ce qu'Aurélia Gaillard regroupe sous le terme de « fabuleux ${ }^{3} »$;

- une transculturelle, puisque le conte se décline indifféremment comme genre populaire ou lettré, païen ou chrétien ; 
- une qui englobe la diversité des publics, dans la mesure où le conte peut s'adresser aussi bien aux enfants qu'aux adultes (naguère appelées " grandes personnes »);

- une perspective transesthétique enfin, puisque le conte s'accommode de toutes les variétés de registre - «tragique » et « comique » - et de tonalité - « naif », « lyrique », « ironique », « humoristique ».

3 À quoi s'ajoute une autre difficulté si l'on considère que la poésie répond non seulement à des critères de fiction, dans la lignée aristotélicienne, mais aussi à des critères de diction (Gérard Genette ${ }^{4}$ ). Ainsi l'usage du vers, qui a largement dominé les poétiques classiques, a progressivement cédé la place à la prose.

On s'interroge donc ici, en couvrant un empan chronologique qui va de la deuxième moitié du XVII ${ }^{e}$ siècle à la fin du XIX ${ }^{e}$ siècle, sur ce que peut signifier le terme de "poésie " appliqué au conte, de fées ou merveilleux, voire fantastique ${ }^{5}$. En d'autres termes, dans quelle mesure le passage d'une poétique classique à une poétique moderne déplace-t-il les enjeux? Doit-on sur ce plan opposer le "classique» La Fontaine - auteur de fables et de contes en vers - au « moderne » Perrault, qui écrit des contes en vers et en prose ? À l'examen, on verra se dessiner en diachronie des phénomènes de permanence, d'évolution ou de transition; et en synchronie, on verra comment les œuvres jouent de l'hybridation et participent à la définition moderne de la prose littéraire. Si les oppositions entre une supposée modernité et ce que l'on nomme par contraste "poétique classique " apparaissent beaucoup moins tranchées que ce que l'on aurait pu croire, on sera néanmoins sensible, à partir du romantisme et jusqu'au symbolisme, aux moyens et à l'imaginaire dont se dotent les contes en prose pour atteindre - quand même - à la poésie comme valeur par excellence, bien qu'elle apparaisse aux yeux de leurs auteurs comme en péril - sinon déchue -, car renvoyant à un passé prestigieux mais révolu de la littérature, tour à tour susceptible de susciter ironie et nostalgie. Le Baudelaire du Spleen de Paris - recueil qui fait aussi la place aux contes de fées et contes merveilleux ${ }^{6}$ - le savait déjà ; mais aussi avant lui Aloysius Bertrand, Xavier Forneret ou Gérard de Nerval ; et après lui, Jules Laforgue, Marcel Schwob ou Jean Lorrain - pour ne citer que quelques noms et jalons de conteurs importants que l'on retrouvera, ou non, dans les pages qui suivent et qui ne prétendent aucunement à l'exhaustivité. Car il n'est point d'autre condition pour refonder la poésie - heur et malheur de la modernité - que de dresser le constat préalable et douloureux d'une certaine mort de la poésie.

\section{Conte - merveilleux - en vers égale poésie ?}

Il y a une sorte d'évidence moderne, mais trompeuse, à associer à la poésie le conte, et en particulier le conte de fées ou merveilleux, car l'évidence tient aussi de la gageure. De façon assez simple, ou en bonne poétique aristotélicienne, l'évidence reviendrait à associer en régime classique un sujet élevé - pour dire vite, les destinées de princes et de princesses -, à un moyen qui l'est également, le vers. Or, la réalité des pratiques et des textes contredit largement une telle appréciation. Jouant des moyens complémentaires des vers et de la prose, déjà La Fontaine et Perrault, dans la seconde moitié du $\mathrm{XVII}^{\mathrm{e}}$ siècle, mettent en œuvre une conception de la poésie beaucoup plus riche et plus subtile que celle qui réclamerait le vers comme moyen. On le saisit intuitivement pour Perrault, qui a doté le patrimoine littéraire français du plus célèbre recueil de contes, successivement avec trois «contes en vers» en 1695 - dont 
l'appellation tardive est apocryphe -, puis avec ses Histoires ou contes du temps passé avec des moralités (1697), cette fois écrits en prose (quoiqu'avec des moralités en vers). Encore l'usage initial du vers, s'il signe le lettré, n'implique-t-il pas chez lui, bien au contraire, une poétique liée à la hauteur ou à la grandeur du sujet. C'est peut-être même la distorsion et la distance qui font le sel du jeu ainsi mis en place. Il y a là, ainsi que le montre Jean-Paul Sermain, comme une étape préparatoire à la poétique des contes en prose où Perrault trouvera sa manière la plus originale et la plus moderne, mais sans pourtant le céder en rien sur la poésie qui, avec lui, se réinvente en quelque sorte génialement dans la prose.

6 Rien de commun, apparemment, avec un sujet mythologique et noble s'il en fut, Les Amours de Psyché et de Cupidon (1669), « idylle héroïque » que La Fontaine avait mise en scène quelque trente ans auparavant; si ce n'est que, jouant sans le savoir à fronts renversés avec son illustre successeur, La Fontaine prenait le parti, surprenant pour un tel sujet, d'" une fable contée en prose ", ou plus exactement du prosimètre, par une conscience symétrique que même le vers ne suffit pas à l'expression développée d'un sujet élevé (" nos vers lassent à la longue ») et qu'il lui faut inventer et approprier pour son conte - qui n'en est pas moins un poème - des moyens d'expression variés. À cet égard, Benjamin Bouchard fait clairement voir à quel point cet "Ancien " que voulut naturellement et principalement être La Fontaine sut aussi être à sa manière un "Moderne ». Dès lors, si, même au XvII ${ }^{\mathrm{e}}$ siècle, le vers ne suffit pas à définir la poésie - cela, Aristote l'affirmait déjà au seuil de La Poétique -, il semble de façon nouvelle que non seulement la prose ne l'interdise pas par principe, mais encore qu'elle puisse la favoriser - tout cela chez des auteurs qui ont non seulement une conscience aiguë de la portée esthétique mais aussi, au moins chez Perrault, de la portée stratégique de leur pratique. C'est pourquoi la vogue du conte en vers au $\mathrm{XvIII}^{\mathrm{e}}$ siècle à laquelle s'intéresse Stéphanie Bernier-Tomas relève d'une tout autre conception de la poésie : pièces de circonstance, joliment dites «fugitives » à l'époque, ces contes légers et volontiers licencieux sont d'abord le fruit d'une sociabilité particulière qui se déploie volontiers avec l'ingéniosité de l'hétérométrie, le brio ou la virtuosité de l'image : tout un art, mineur certes, non pas négligé mais en négligé, volontiers décrié en son temps et ignoré ensuite, mais qui, selon l'auteur de l'article, tient à la poésie par son traitement de l'image et par son caractère d'" expérimentation verbale ", de savant désordre, ou encore, sous des allures policées, de subversion des mœurs et du discours en faveur d'une morale et d'une esthétique de la liberté - faut-il dire du libertinage ? On voit que la définition de la poésie ne tient pas ici, sinon de façon purement tautologique, à l'usage du vers, mais bien davantage à ce que celui-ci rend possible par sa variété et sa plasticité, fût-ce apparemment à contre-emploi : si noblesse oblige, roture, même et surtout en vers, donne licence.

7 Si donc l'usage du vers ne suffit plus à définir la poésie des contes, et si la poésie n'équivaut pas - ou plus - à l'usage du vers, qu'est-ce donc que la poésie des contes ? Le $\mathrm{XIX}^{\mathrm{e}}$ siècle, on le sait, va achever de bouleverser un système nommé après coup "classique », et dont on vient d'entrevoir qu'il n'était aussi assuré que par la décision d'une modernité autoproclamée qui le constituait en commode repoussoir. Notre gageure pourtant reste entière, car que faire du conte dès lors que la poésie s'autonomise comme genre, voire comme genre non narratif en France à la fin du XIX siècle ? et qui plus est, avec ce nouveau genre poétique en prose qu'est le poème en prose, peut-être appelé à devenir le genre poétique moderne par excellence ${ }^{7}$ ? 


\section{Du merveilleux des contes poétiques en prose ${ }^{8}$}

8 S'il n'est de poésie sans moyens, vers ou prose, celle-ci - en régime romantique et moderne - va donc désormais s'apprécier davantage comme effet, c'est-à-dire comme style, et plus encore peut-être comme imaginaire et comme valeur. On sait à quel point le romantisme allemand, notamment avec les frères Grimm, va promouvoir l'idée d'une poésie née du peuple (Volkspoesie) par opposition à une poésie d'auteurs savante et lettrée (Kunstpoesie), même si, comme le suggère Natacha Rimasson-Fertin, le parti pris explicite en faveur de la première n'est adopté qu'au prix d'un fort paradoxe : du statut de simples collecteurs, les frères Grimm passent à celui d'adaptateurs et pour finir, bel et bien à celui d'auteurs. Plus largement, avec le motif du revenant dans Ondine, un conteur-poète génial comme La Motte-Fouqué écrit un « récit hautement littéraire qui met en scène des croyances populaires et une conception magique du monde ». Et encore au-delà, l'imaginaire romantique subsume une conception du conte et une conception de la poésie exhaussés en valeurs dont les sources populaires ne sont plus que le truchement.

9 À cet égard, l'originalité, voire l'unité des Contes et facéties (1852) de Gérard de Nerval, dont Jean-Nicolas Illouz parvient à cerner malgré tout la poétique diverse et subtile - mais également singulière, et je me risquerai à dire " pivotale » - procède justement d'une manière variée dans l'esprit et dans le ton qui conjoint ironie et merveilleux au sein du recueil, tout en relevant d'une conscience aiguë et angoissée de la place désormais improbable et paradoxale du poète-conteur dans un monde désenchanté, dès lors que, pour l'auteur des Petits Châteaux de Bohême (1853) et des Filles du feu (1854) déjà - et une petite dizaine d'années avant Baudelaire -, « la poésie [est tombée] dans la prose $^{9}$ ». On mesure à quel point on s'est éloigné de la manière et de l'esprit joyeusement léger des contes en vers du siècle précédent. C'est que la situation historique et le cercle métaphysique ne sont plus les mêmes, ni l'esthétique. L'opposition partiellement technique du vers et de la prose est dès lors devenue une question lourde, ou grave, c'est-à-dire de vision, ainsi que l'affirmera Proust à propos du style.

Quelque trente ans après Nerval, le Jules Laforgue des Moralités légendaires (1887) est l'héritier d'une situation de la poésie que le contexte historique, sa situation personnelle et son idiosyncrasie de conteur-poète portent à l'extrême de la tension et du paradoxe. Après Les Complaintes (1885), les Moralités sont une façon extrêmement élaborée pour mettre en œuvre, selon l'heureuse formule d'Henri Scepi, "une fabulation de la poésie » qui poursuit l'exploration des voies idéales de l'art par d'autres moyens, dès lors que le lyrisme poétique est devenu hautement suspect. Les «moralités » (en vers) dont Perrault accompagnait ses contes ne sont pas ici dissociables des contes eux-mêmes. Non plus incertaines et ambiguës, elles ne peuvent être désormais que perverses - sur le plan moral - et parodiques - sur le plan intertextuel -, encore qu'elles aient surtout pour fonction de réorienter la poésie vers un chemin nouveau: il n'y a certes pas d'autre merveilleux, mais c'en est un. Or les " contes », parfois aussi appelés "nouvelles » par Laforgue, ne le sont précisément que par un acte du poète auquel le lecteur est invité à souscrire. Les sources du "légendaire » y sont en effet fort hétérogènes - mythologie païenne antique avec «Persée et Andromède » ou "Pan et la Syrinx »; mythologie biblique avec "Salomé », 
et chrétienne avec "Le Miracle des roses ", qui recycle à sa manière, entre autres sources, le récit hagiographique de la vie de sainte Élisabeth de Hongrie, et fait un peu figure d'intrus ; fonds mythologique enfin, emprunté à Shakespeare pour " Hamlet ", à Wagner pour "Lohengrin ». Tout aussi hétérogènes sont les réalisations textuelles considérées, bien qu'elles aient en commun d'être des palimpsestes de tradition ancienne - ce qui vaut certes pour les contes -, mais aussi des indices de culture très savante puisant à de multiples sources. À quoi s'ajoute une efflorescence d'époque qui peut aller jusqu'à saturation: on pense au mythe de Salomé. Surtout, au mépris de toute essentialisation, le «conte» relève bien, pour parler comme Jean-Marie Schaeffer, d'une "intentionnalité pragmatique ${ }^{10}$ " partagée en amont et en aval par l'auteur et le lecteur. Déconstruit, démythifié, le légendaire est ainsi soumis au fort travail de sape de la parodie qui relève chez Laforgue d'une hypersensibilité métapoétique articulée à une philosophie de l'art puisée dans La Philosophie de l'Inconscient (1880) de Hartmann (1842-1906), disciple de Schopenhauer pour qui l'art concentre et réfléchit la création tout entière.

11 À ce versant somme toute positif s'oppose le versant nettement plus contrasté et ambivalent de l'Inconscient, expression de la Nature comme force vitale majeure, instinctive et irrépressible, qui implique l'acceptation de la vie jusqu'à une absolue réconciliation avec elle - sur ce point le disciple rompt avec le maître. De manière plus sombre mais non moins nécessaire, l'Inconscient de Hartmann est un matérialisme qui tord le cou aux croyances rassurantes de l'idéalisme sur les possibilités de connaissance et de maîtrise de soi du sujet pensant : parallèlement à l'œuvre de Nietzsche dont il est l'exact contemporain (et qui sera son féroce critique), c'est là une machine de guerre lancée contre l'ontologie et la métaphysique. Or, en tant que principe supérieur d'une philosophie de la négativité, l'Inconscient est revendiqué sans faille ni relâche par Laforgue comme la grande intuition spéculative dont son œuvre propose le pendant et la traduction esthétique. Ainsi, la voie de l'Inconscient, « Loi » unique, commande aussi une poétique. En ce sens, les Moralités en prose poursuivent un cheminement parallèle à celui de la libération du vers et de l'emploi du vers libre en 1886-1887, qui affranchissent le poète lui-même des anciens carcans en le conduisant à inventer de nouvelles solutions formelles. Le lyrisme s'y déploie en se détachant du sujet - jamais peut-être "la disparition élocutoire du poète " évoquée par Mallarmé n'a reçu plus adéquate illustration - en traduisant l'élan d'un désir dépersonnalisé et, si l'on ose dire, dé-lyricisé. Mais c'est ainsi seulement, selon les exactes formules d'Henri Scepi, que peut se fonder la « repoétisation » comme « un geste de réappropriation de la valeur de la poésie ». Pour un matérialiste en quête d'idéal comme Laforgue, il ne s'agit de rien de moins que de réinventer la langue, "à la fois savante et virginale, pure et raffinée " dans l'espoir, in fine et malgré tout, de faire renouer la poésie avec le frisson de l'absolu. L'affirmation ne va pas sans paradoxe : il faut comprendre qu'ironie et parodie ne sont pas pure négativité, mais les étapes d'un processus. Car le poète moderne, au prix d'une ascèse comparable à la démarche dont Mallarmé fut l'initiateur, sait que la poésie est ce rien avec lequel il doit faire œuvre pour le conduire vers l'enchantement de l'acte poétique : c'est lui désormais qui offrira seul les prestiges de la fiction dans un monde désenchanté, c'est-à-dire privé de transcendance - métaphysique ou religieuse -, et même du recours aux puissances païennes des fées et du merveilleux.

12 Chez Schwob également, célébré par ses pairs comme l'écrivain le plus savant, le plus érudit et peut-être le plus doué de sa génération, on trouvera une conscience métapoétique hyper-aiguisée que l'écrivain reverse au travail de la poésie elle-même, 
encore que d'une manière très différente de celle qui prévaut chez Laforgue. À cet égard, les préfaces des recueils sont des textes importants et profonds, mais aussi des dispositifs stratégiques complexes et retors, voire pervers. Qu'en est-il chez lui du conte poétique en prose ? À rebours de ce que nous avons pu observer chez Laforgue, s'imposent chez l'auteur du Roi au Masque d'or (1892) une philosophie idéaliste en rupture manifeste avec le matérialisme positiviste du temps, et avec elle une esthétique qui ambitionne d'inventer un nouveau réalisme littéraire, lequel prendra le contre-pied du naturalisme ambiant : nouveau réalisme du singulier, du contingent, de l'individuel, de l'impression, voire « impressionnisme » dans la mesure où l'artiste se doit, selon ses propres mots, d'« imiter la nature dans les formes que nous saisissons en elle ». Mais Schwob va plus loin, lui qui a été à l'école de Michel Bréal (1832-1915) et de son plus fameux disciple, Ferdinand de Saussure (1857-1913), en articulant sa philosophie avec une prescience linguistique moderne du signe. Celle-ci relève en effet d'une " conception [...] sémiotique du réel», selon la formule de Pauline Bloch et Michel Viegnes, laquelle prend déjà le parti, dans la terminologie que développera plus tard le linguiste et critique Michael Riffaterre, d'une semiosis illimitée : «Sachez que tout en ce monde n'est que signes, et signes de signes. " Encore ne faut-il pas couper une telle abstraction potentielle de ce qui la fonde au cœur de la perception schwobienne du monde, et qui s'inscrit également au cœur de sa poétique : l'impression, avons-nous dit, trouve certes son principe dans la sensation, c'est-à-dire ce qui affecte le corps, mais qui trouve son point d'aboutissement - ou son effet - dans l'émotion que doit susciter l'œuvre d'art. Point de contradiction donc entre la «symétrie» de la composition, entendue selon l'étymologie comme la loi même de l'esthétique, et l'intuition saisissante, bouleversante, voire sidérante qui se dégage à la lecture des contes. À cet égard, l'usage de l'image joue un rôle déterminant, parce qu'elle suscite ou plus proprement crée des singularités qui échappent à l'ordre de l'expérience commune - on n'est pas si loin du "stupéfiant image » des surréalistes, mais ici surgi d'une pratique réglée de la prose. Dans l'univers fascinant de Schwob, tout est pris dans une dynamique de la singularité et du double, autant dire du masque, qui dérive potentiellement vers le multiple et qui, dans la conscience moderne, ne recouvre peut-être pas de visage. Chez le Jean Lorrain des Histoires de masques (1900), ce sera l'angoisse fondamentale. Mais dans l'univers de Schwob, la mise en œuvre des masques - clairement thématisés par exemple dans "Le Roi au masque d'or ", «La Peste », ou "Les Faulx-Visaiges »ressortit plutôt à une conception inquiète, certes, mais néanmoins perçue comme féconde en ce qu'elle permet d'appréhender, par le truchement des images, la diversité des phénomènes (de ce qui apparaît) sous lesquels le réel se donne à lire et à percevoir. Cela vaut bien entendu au sein de chaque conte, mais aussi d'un conte à l'autre dans l'espace du recueil (chez Laforgue en revanche, chaque conte poursuit un idéal poétique, mais les Moralités dans leur ensemble ne peuvent guère se prévaloir d'une poétique du recueil).

13 Au-delà, comme on le verra dans les exemples analysés, l'image s'insinue dans le conte - qui relève ici d'une poétique du récit bref - comme une création originale de la prose. Liée au corps en tant que vecteur d'émotions et de souffrances - les tourments et les supplices ne manquent pas -, l'image le donne aussi à lire comme surface, lieu en somme où s'inscrivent la trace, le signe et le sens, ce dernier toujours fuyant et à interpréter. Centre des interactions, elle est aussi, comme le soulignent Pauline Bloch et Michel Viegnes, "mémoire du passage d'autrui » et "signature individuelle»: singularité, donc. Mais au risque d'insister sur une évidence, il faut ajouter qu'elle est 
imposée par une autre singularité, celle du style de son auteur. À cet égard, Schwob, dans sa préface à " "La tragique histoire d'Hamlet" de William Shakespeare » - objet d'un intérêt partagé avec Laforgue et d'autres écrivains de la fin de siècle -, établit très consciemment et très clairement la prose et la poésie - les vers - comme moyens alternatifs de la traduction du monde, bien que non rivaux. Cela ne l'empêche pas, en moderne, d'assigner quant à lui - et non sans paradoxe - «la gloire du verbe ${ }^{11}$ » à la prose. Comprenons que la poésie, mode d'expression et de création esthétiques, est une interprétation, un point de vue sur le réel, et nullement un moyen - prose ou vers - ou une forme a priori.

La dernière singularité sur laquelle il convient de mettre l'accent est celle d'une esthétique de la réception très concertée, située dans un cadre herméneutique où il n'est plus permis d'embrasser la Vérité - Nietzsche n'est pas loin -, et où il appartient au lecteur à son tour de se doter d'un point de vue créateur. L'imagination est alors confrontée à ce que Schwob nomme les "silences du récit». Sa démarche n'est pourtant pas tout à fait déjà, comme on le devine, celle d'un Wolfgang Iser théorisant un siècle plus tard "l'acte de lecture ${ }^{12}$ ", avec laquelle elle semble résonner ici étrangement. L'interprétation créative chez Schwob est en effet toujours à vif, jusque dans ses béances, la prégnance des images créant une différence de potentiel, comme on le dit d'un courant électrique, avec la poétique du récit bref. C'est pourquoi, si l'on n'imagine guère de lecteur des contes en prose de Schwob qui ne soit aussi un lettré ouvert aux spéculations sur les grandes questions modernes de la philosophie et de la littérature, le lecteur insensible aux pouvoirs de l'imaginaire peut passer son chemin. On sait à quel point Schwob a été une source féconde pour Borges, et on comprend qu'il l'ait été. Mais il est permis de préférer chez le premier, comme une émanation singulière de l'imagination créatrice combinée à l'intelligence, la capacité toute particulière de créer des images qui hantent.

Avons-nous affaire à une poétique davantage en retrait, aujourd'hui plus datée, avec les « contes-bibelots » de la fin de siècle et tout particulièrement les Princesses d'ivoire et d'ivresse (1902) de Jean Lorrain? Conjoindre l'art du verre et l'art du verbe sous le signe de la miniature, voilà une tentation très fin de siècle en effet, qu'on pourrait juger décadente si elle ne visait qu'à figer la nature en artifice, au mépris de la vie elle-même. Un certain « devenir-bibelot de la littérature » (Bernard Vouilloux), chez les Goncourt par exemple, pourrait aller en ce sens. Certes, comme l'écrit Cyril Barde, «la métaphore topique de l'écriture-bijou, de l'écrivain ciseleur semble ici remotivée par Lorrain ». Mais il ne faut pas s'y tromper, et l'on ne saurait mieux dire que l'auteur de l'article à propos du plus illustre maître verrier contemporain : «La poétique de Gallé relève elle-même d'un double processus de métamorphose qui enchante la matière en lui insufflant l'Idée et le rythme de la vie organique." Ainsi, sous le signe du symbolisme puis de l'Art nouveau, un grand artiste tel que Gallé, qui se veut poète et sera célébré comme tel par les écrivains de sa génération, cherche non à contenir et à enfermer une nature figée, ou morte, mais à la quintessencier et à la spiritualiser en poésie : à créer, en somme, ce que le poète anglais Keats a nommé " a thing of beauty ", laquelle, au risque d'un anachronisme, relève, plutôt que de la miniature proprement dite, de ce qu'André Breton nommera un peu plus tard " signe ascendant ».

16 Si l'univers de Gallé apparaît proche des contes, c'est donc que son œuvre de cristal est inspirante au point d'en évoquer les talismans et les enchantements (non de suggérer des fétiches). De façon symétrique, pour le meilleur et pour le pire, les contes de 
Lorrain ont pu être rapprochés de l'art du verrier. Au-delà de l'autonomie et de la condensation propres au récit bref, les récits semblent immobilisés, gelés, placés sous verre, en somme bloqués par leur tropisme décadent qui s'accorde à la froideur et à l'égoïsme de ses personnages. Là encore, c'est une question d'images - qui mettent en série neige, givre, glace et verre -, et finalement de style. Mais pour bien comprendre la portée de l'équivalence entre le conte poétique en prose et la verrerie, selon un trajet qui conduit du premier à la seconde - du comparé texte au comparant bibelot -, il ne faut pas oublier que c'est d'abord Gallé lui-même qui invente et théorise la "verrerie parlante ", laquelle désigne à ses yeux un objet proprement poétique serti de citations en vers ou de poèmes gravés, marquetés, voire enclos dans le verre. Le symbolisme postwagnérien eût dit « art total » - avec la poésie, la musique est également présente ; nous dirions aujourd'hui «art intersémiotique ». Car il ne s'agit pas là d'illustrations naïves, mais bien plutôt de tentatives originales qui réalisent le devenir-poème des créations du maître verrier - et ses contemporains, critiques et artistes, ne s'y sont pas trompés. L'équivalence de la matière et de la parole, toutes deux transcendées en vue d'une unité harmonieuse supérieure, a dès lors pour fonction de montrer que, en régime symboliste, la poésie, idéal et valeur, est l'art par excellence qui subsume tous les autres. Or chez Gallé, la création du poème de verre est pleinement accomplie. Il n'empêche, comme le montre Cyril Barde, qu'il est bien un devenir-bibelot de certains contes de Lorrain, mais selon une signification autrement plus riche que celle qui expose, avec les "perversions fin de siècle du merveilleux ${ }^{13}$ ", les symptômes d'une œuvre décadente. Il s'agit alors, pour le conteur-poète, d'instituer le verre comme élément artificiellement et esthétiquement protecteur d'une magie précieuse mais condamnée, celle précisément des contes liés au monde disparu de l'enfance et de la croyance. C'est là qu'on trouve le meilleur de Jean Lorrain, dans une nostalgie poétique fort émouvante qui peut rappeler Nerval, et qu'on aurait tort de situer en fin de parcours comme si fin de siècle valait ici pour fin de race (littéraire) - comprenons épuisement des facultés de désir et d'imagination. La Décadence ne vaut que dans la mesure où elle est le théâtre, et parfois le moment, où elle s'affronte à ses propres démons. Assurément Jean Lorrain, comme par exemple Henri de Régnier et plus que Robert de Montesquiou, sut en faire bon usage. Mais il est temps de s'arrêter quelque peu sur cette sempiternelle gageure que serait à ce stade la définition du conte. Nous l'avons dit « merveilleux » : nous le voulons aussi « poétique », en vers ou en prose.

\section{Qu'est-ce qu'un conte merveilleux poétique ? D'une poétique historique résolument anachronique}

17 Faut-il rappeler que la définition même du conte ne va pas du tout de soi pour les textes $\mathrm{du} \mathrm{XVII}^{\mathrm{e}}$ siècle, diversement voire indifféremment nommés "fables", "nouvelles ", " contes", « histoires» - ne serait-ce que chez Perrault, excusez du peu! - et que, a posteriori, la tradition a reconnus comme tels? Mais l'anachronisme raisonné peut aussi avoir ses vertus. Ne craignons donc point de prendre du champ, et méditons avec Milan Kundera sur l'essence historique de "l'art du roman ", la réflexion pouvant être reconduite à profit pour le conte. Je cite ici un peu longuement l'auteur des Testaments trahis :

Pour pouvoir former un seul tout au cours des siècles cette histoire [celle du roman] ne doit-elle pas être unie par un sens commun et, donc, nécessairement suprapersonnel ? Non. Je crois que même ce sens commun reste toujours personnel, 
humain, car, pendant la course de l'histoire, les concepts de tel ou tel art (qu'est-ce que le roman ?) ainsi que le sens de son évolution (d'où vient-il et où va-t-il ?) sont sans cesse définis et redéfinis par chaque artiste, par chaque nouvelle œuvre. Le sens de l'histoire du roman c'est la recherche de ce sens, sa perpétuelle création et re-création, qui englobe toujours rétroactivement tout le passé du roman ${ }^{14}$. relativiste qui refuse les catégorisations et les classements au profit d'appréciations singulières indépassables, presque à la manière d'un Benedetto Croce: à chaque romancier, donc, voire à chaque roman sa poétique, dont ne saurait rendre compte aucune tentative de rationalisation scientiste, voire de système transcendant. Aussi la dernière phrase semble-t-elle aboutir à une conception, certes dynamique mais minimale, de "l'histoire du roman » comme "perpétuelle création et re-création ». Mais celle-ci comporte un ajout de taille, presque une hyperbate - [création et recréation] « qui englobe toujours rétroactivement tout le passé du roman »-, cette fois assurée et passablement péremptoire, dont on voit qu'elle relance et réoriente complètement la réflexion vers le point souhaité de la démonstration :

Rabelais n'a certainement jamais appelé son Gargantua-Pantagruel roman. Ce n'était
pas un roman; ce l'est devenu au fur et à mesure que les romanciers ultérieurs
(Sterne, Diderot, Balzac, Flaubert, Vancura, Gombrowicz, Rushdie, Kis, Chamoiseau)
s'en sont inspirés, s'en sont ouvertement réclamés, l'intégrant ainsi dans l'histoire
du roman, plus, le reconnaissant comme la première pierre de cette histoire ${ }^{15}$.

Pastichons Kundera : s'il n'était pas sûr que Perrault eût écrit des " contes » - plutôt que des « histoires" par exemple - et moins encore La Fontaine un "conte » avec Psyché, etc., ceux-ci le deviendraient en vertu de la tradition qui les a accueillis comme tels, et au moins pour les premiers, constitués en pierre fondatrice de cette histoire. Pour le dernier, on peut même s'amuser à penser qu'il l'est définitivement devenu grâce à la contribution de Benjamin Bouchard et par sa place dans le présent volume. Si la question était d'emblée résolue pour $\mathrm{M}^{\mathrm{me}} \mathrm{d}$ 'Aulnoy, toujours identifiée comme " conteuse»-sans que l'autre question, celle de la définition du conte de fées qui supposerait un introuvable modèle, puisse trouver de réponse définitive, comme le souligne prudemment Philippe Hourcade -, elle semble plus assurée ensuite : conte en vers du $\mathrm{XVIII}^{\mathrm{e}}$ siècle; contes de fées ou merveilleux du romantisme allemand et européen; et jusqu'aux recueils de contes $d u \mathrm{XIX}^{\mathrm{e}}$ siècle français ${ }^{16}$, qui démarquent ostensiblement dès leurs titres les modèles $d u$ XVII $^{\mathrm{e}}$ siècle - qu'il s'agisse des Contes $d u$ rouet (1885) ou des Oiseaux bleus (1888) de Catulle Mendès -, ou qui s'en éloignent subtilement du côté d'une littérature hautement raffinée, mais n'en revendiquent pas moins une filiation merveilleuse, fût-elle imaginaire, à l'instar des Princesses d'ivoire et d'ivresse (1902) de Jean Lorrain. Entre temps, les catégories ont pu se brouiller passablement, comme c'est déjà le cas chez Nerval, mais plus encore chez Laforgue ou chez Schwob, pour lequel la notion de «conte " ne saurait correspondre à une essence des textes (ou du moins de tous les textes), mais à une appellation insistante que problématise l'espace du recueil ${ }^{17}$. C'est ainsi que d'une position antirationaliste et non historicisante a priori, la réflexion en vient à poser les bases d'une poétique historique anachronique et rétrospective, car en quelque sorte cumulative. Mais ainsi que le suggérait Kundera, l'admission a posteriori dans le genre n'interrompt pas l'arraisonnement (potentiellement sans fin), dans la mesure où celui-ci est constitutif de la poétique du genre. 


\section{Contes merveilleux en vers et/ou en prose : pour une poésie intégrative}

20 Assurément, avec le regard rétrospectif que peut porter le XIX ${ }^{\mathrm{e}}$ siècle sur l'âge classique, la poétique du conte a connu des transformations décisives. Plutôt que d'évolution(s), faut-il parler de révolution en vertu d'un changement de paradigme? Peut-être, à condition d'admettre que la situation esthétique des œuvres dans l'histoire ne s'inscrit pas dans la trajectoire purement linéaire d'une vision finaliste - qu'on n'aura garde de confondre avec la distribution chronologique des articles ici réunis-, laquelle commanderait une marche forcée vers ce point complaisant de la "modernité " où nous croyons nous-mêmes nous situer, annexant au passage un nom de genre à géométrie variable dont la flottante définition serait compensée par une validation axiologique opérée plus ou moins par la bande. Bien sûr, la valorisation du fonds populaire par le romantisme allemand comme source de poésie contribue à infirmer la définition de la poésie par le "moyen» du vers. Mais elle ne doit pas faire oublier la démarche du « Moderne » Perrault, qui anticipe de façon très concertée ce changement en légitimant le genre des contes - en vers puis en prose - de deux manières. En arguant de leur vérité et de leur moralité supposées, Perrault fait valoir leur supériorité sur les fables antiques, réputées tout à la fois mensongères et immorales. Mais il fait aussi coup double. Car en affirmant s'inspirer des contes de nourrices (donc d'origine populaire) "[rapportés] naïvement en la manière qu'il les a ouï conter dans son enfance ", il privilégie le naturel et prend ses distances à l'égard des contes galants et hautement lettrés de $\mathrm{M}^{\mathrm{lle}}$ Lhéritier ou $\mathrm{M}^{\mathrm{me}} \mathrm{d}^{\prime}$ Aulnoy, tout en prétendant destiner ses contes aux enfants qu'il s'agit d'instruire et d'édifier. La question du « moyen » devient dès lors seconde, ou plutôt sa manière en prose sert désormais la cause de Perrault. Parallèlement, on sait aussi que, dès le XVII siècle, la question de la prose est posée avec les grands genres que sont la tragédie et l'épopée, même si cette prose est encore à bien des égards transgressive ou incongrue au regard de ce que l'on a appelé depuis la " poétique classique ». Sans parler du roman, voire du drame en prose, le "poème en prose » ne sera qu'un des avatars - certes remarquable - de la longue histoire de la poésie en prose ${ }^{18}$, et il croise comme on l'a vu notre conte poétique en prose. Pourtant, Aloysius Bertrand n'est pas, avec Gaspard de la nuit (1842), le premier ni le seul auteur de " poèmes en prose ». À cet égard, sa consécration par Baudelaire en vertu d'un geste inaugural d'instauration du genre par réappropriation - geste qu'entérineront à sa suite les générations suivantes - tient notamment à ce qu'il s'agit bien du premier recueil de poèmes en prose revendiqué comme tel.

21 On a vu pourtant que nombre des contributions qui vont suivre se proposent d'interroger la poétique du conte merveilleux et/ou de fées en affrontant en toute rigueur la question du «moyen » (vers ou prose) à celle du « sujet » : cela sur toute la période considérée, sans que cette double question disparaisse dès lors que cesse, au moins de manière significative, la pratique du conte en vers après le XvIII ${ }^{e}$ siècle. Il est néanmoins possible de s'affranchir de la difficulté pour aborder la poétique des auteurs et des œuvres, en laissant en arrière-plan le choix du vers ou de la prose. Et c'est à l'origine, fût-elle mythique, entendons au XVII siècle, que l'interrogation, sans être moins pertinente en termes de stratégie d'écriture, est peut-être moins contraignante en termes d'imaginaire, comme si le problème surgissait vraiment au xix ${ }^{\mathrm{e}}$ siècle avec la liquidation programmée du vers comme médium poétique. Le cas Perrault - en vers 
puis en prose - pourrait bien en fournir la preuve sur les deux versants dudit problème, tout comme celui de $\mathrm{M}^{\mathrm{me}} \mathrm{d}$ 'Aulnoy, résolument en prose - mais sans que la conteuse s'interdise au besoin de recourir au vers. Encore Perrault, dans ce conte en prose qu'est «La Barbe bleue » et que commente Pierre-Emmanuel Moog, suspend-il l'intérêt du conte à la fameuse question réitérée - «Anne, ma sœur Anne, ne vois-tu rien venir ?»-, en somme à un alexandrin avec césure lyrique (sur « e » muet) dont l'efficacité poétique est assez prouvée par la tradition - et qui impose son évidence au sens de la fonction poétique de Jakobson. C'est dire que le conte poétique en prose sait aussi jouer de l'alternance de la prose et du vers au bénéfice du style, et du rythme. Or si "vers il y a sitôt que s'accentue la diction, rythme dès que style ", comme le veut généreusement Mallarmé dans "Crise de vers» (1886-1892-1896), lui pour qui «la forme appelée vers est simplement elle-même la littérature ", on peut bien entendu - et ce n'est pas le moindre paradoxe - trouver une application à la formule bien avant la fameuse «crise ». Perrault prosateur le prouve superbement. $\mathrm{M}^{\mathrm{me}} \mathrm{d}^{\text {'Aulnoy }}$ n'est sans doute pas en reste, qui réaménage à sa manière le conte de fées en prose en usant d'une liberté tous azimuts. Assurément, le genre devient chez elle tout aussi bien conte (en prose) que poésie (indépendamment du vers) grâce à la capacité d'affranchissement, de renouvellement, d'invention et d'imagination de l'illustre conteuse. On perçoit certes chez elle une poétique de l'excès qui n'a rien de classique et, par-dessus tout, ce qu'il faut peut-être appeler de la verve ${ }^{19}$. Peu importe que le conte de fées n'y trouve point son secret ou sa formule; et si Philippe Hourcade tient à prévenir tout anachronisme dans l'attribution d'une douteuse "modernité » de principe à $\mathrm{M}^{\mathrm{me}} \mathrm{d}$ 'Aulnoy, on ne peut s'empêcher de penser que, malgré qu'il en ait, c'est bien en moderne qu'il la lit. Mais où commence la modernité ?

À lire les articles qui suivent, on se persuadera peut-être, ce qui serait fort rassurant, que les catégories commodément reçues de la poétique historique demandent à être réaménagées avec quelque finesse, et non moins de souplesse. Dès le XVII siècle en effet, le ver de la prose a déjà pénétré dans le fruit du vers. Dans un autre sens que le constat de Mallarmé, qui peut sonner comme le pendant esthétique du «Dieu est mort » proclamé par Nietzsche dans Le Gai Savoir (1885), il apparaît clairement qu'au XVII ${ }^{\mathrm{e}}$ siècle déjà, « on a touché au vers ».

\section{NOTES}

1. Il s'agit paradoxalement d'une définition du conte qui figure dans un roman de l'auteur, le cinquième, intitulé Être ou ne pas être (1857) : voir H. C. Andersen, Contes et histoires, éd. intégrale des textes rassemblés par l'auteur, introduction, trad. et annotation par M. Auchet, Paris, Librairie générale française, Le Livre de poche, coll. « La Pochothèque », 2005, p. 29.

2. J.-M. Schaeffer, Qu'est-ce qu'un genre littéraire ?, Paris, Seuil, coll. « Poétique », 1989.

3. A. Gaillard, Fables, mythes, contes. L'esthétique de la fable et du fabuleux (1660-1724), Paris,

H. Champion, coll. « Lumière Classique », 1996.

4. G. Genette, Fiction et diction, Paris, Seuil, coll. « Poétique », 1991. 
5. Ces distinctions reçues et fort pertinentes, que l'on pourra observer au fil des analyses, importent peu en revanche pour la position générale du problème : sans entrer dans le détail, nous retiendrons plutôt ici la catégorie du «merveilleux » comme la plus large et susceptible d'accueillir toutes les autres.

6. Voir à ce sujet ce que Michel Viegnes explore de façon circonstanciée comme les «dérives diégétiques du poème en prose" - communication non publiée -, dont je tente de rendre compte dans mon Poète, même en prose, Paris, Presses universitaires de Vincennes, coll. «L'Imaginaire du Texte », 2010, p. 47-54. Bien sûr, ces "dérives " ne sont telles que si l'on se fonde sur les attendus récurrents du poème en prose tels que définis selon une tradition qui remonte à S. Bernard, Le poème en prose de Baudelaire jusqu'à nos jours, Paris, Nizet, 1959 et se poursuit jusqu'aux études les plus récentes, lesquelles ne la contestent pas sur ce point : voir par exemple M. Sandras, Lire le poème en prose, Paris, Dunod, 1995 ; N. Vincent-Munnia, Les premiers poèmes en prose: généalogie d'un genre dans la première moitié du dix-neuvième siècle français, Paris, H. Champion, coll. «Romantisme et Modernités », 1996 ; Y. Vadé, Le poème en prose et ses territoires, Paris, Belin, coll. «Sup », 1996 ; ou encore Chr. Leroy, La poésie en prose française du XVII siècle à nos jours : histoire d'un genre, Paris, H. Champion, coll. « Unichamp-Essentiel », 2001. Car pour peu que l'on sorte de ce que sera par la suite l'interdit fait à la poésie de raconter, dont l'exclusive remonte à la fort contestable interprétation de la poétique de Mallarmé imposée par Valéry aux générations futures, il n'y a évidemment pas lieu de dénoncer le récit comme une " dérive ", fûtce dans la poésie moderne. Voir encore sur cette question l'ouvrage classique de D. Combe, Poésie et Récit, une rhétorique des genres, Paris, Corti, 1989.

7. Sur ce point, voir plus haut la note 5 .

8. Je renvoie à cette sorte d'appellation contrôlée que j'ai essayé de fournir dans Poète, même en prose (ouvr. cité) à propos des contes poétiques en prose et, plus précisément, des recueils de contes poétiques de la génération symboliste (1890-1900), ainsi que dans les six recueils de contes symbolistes qui en constituent le principal corpus d'étude, publiés en trois volumes sous ma direction aux ELLUG (Grenoble, désormais UGA Éditions), respectivement en 2009, 2011 et 2016, et consacrés à des recueils de Bernard Lazare, Marcel Schwob, Remy de Gourmont, Henri de Régnier, Georges Rodenbach et Camille Mauclair.

9. G. de Nerval, Petits châteaux de Bohême (1853), "Second château », dans Euvres complètes, éd. J. Guillaume et Cl. Pichois, Paris, Gallimard, coll. « Bibliothèque de la Pléiade », t. III, 1993, p. 419. Par son sous-titre, "Prose et poésie », le recueil signale son contenu selon une opposition reçue qu'il est encore autorisé d'entendre de façon purement formelle, mais qu'il est loisible à chacun de déplacer de manière vagabonde dans l'espace du recueil, puisqu'il s'agit beaucoup moins pour Gérard d'une opposition radicale et tranchée que d'une relation, certes en partie dénouée, mais telle quelle tout aussi nécessaire et féconde, où se situent l'éthos et l'imaginaire pareillement indivisibles $d u$ poète et conteur. À cet égard, je remercie tout particulièrement Jean-Nicolas Illouz d'attirer mon attention sur la dédicace liminaire du recueil « À un ami ». (Il s'agit du même Arsène Houssaye, directeur de L'Artiste puis de La Presse - la poésie est en effet bel et bien d'abord tombée dans la prose de la presse - qui sera le destinataire de la célèbre et fort commentée lettre dédicatoire de Baudelaire: geste stratégique et inaugural du genre qui accompagne la première livraison des Petits Poèmes en prose du futur Spleen de Paris dans La Presse des 26-27 août 1862.) Il me faut citer ici un peu longuement la lettre dédicatoire de Nerval : « Mon ami, vous me demandez si je pourrais retrouver quelques-uns de mes anciens vers, et vous vous inquiétez même d'apprendre comment j'ai été poète, longtemps avant de devenir un humble prosateur. / Je vous envoie les trois âges du poète - il n'y a plus en moi qu'un prosateur obstiné. J'ai fait les premiers vers par enthousiasme de jeunesse, les seconds par amour, les derniers par désespoir. La Muse est entrée dans mon cœur comme une déesse aux paroles dorées ; elle s'en est échappée comme une pythie en jetant des cris de douleur. Seulement, ses derniers accents se sont adoucis à mesure qu'elle s'éloignait. Elle s'est détournée un instant, et j'ai revu comme en un 
mirage les traits adorés d'autrefois !» (p. [419]) Les deux dernières phrases sont d'une beauté à la mesure de leur portée essentielle : le visage de la Muse aimée, disparue et retrouvée, comme est retrouvé le temps chez Proust dont on comprend qu'il fut fasciné par Nerval, voilà qui définit, à travers les chagrins et la consolation, la poétique même de Nerval dans ce qu'elle a de plus esthétiquement émouvant. C'est dire aussi qu'il y a là une forme de symétrie saisissante, mais réorientée de façon heureuse, avec ce qu'écrit Flaubert presque au même moment dans sa fameuse lettre à Louise Colet du 22 juillet 1852 : «Quelle chienne de chose que la prose ! Ça n'est jamais fini ; il y a toujours à refaire. Je crois pourtant qu'on peut lui donner la consistance du vers. Une bonne phrase de prose doit être comme un bon vers, inchangeable, aussi rythmée, aussi sonore. » Certes, c'est le style qui polarise les affres de Flaubert, là aussi au-delà du partage de la prose et du vers; mais surtout, il importe de voir comment chez Nerval, l'héroïque labeur de l'auteur futur de Madame Bovary (1857) se retourne en une rêverie malgré tout apaisée par le fait même qu'elle énonce qu'elle n'en aura jamais tout à fait fini avec la poésie. Ou encore, parce que celle-ci convoque la beauté dans son essence nostalgique qui est celle-là même de la mémoire et du voile : aura d'un visage et d'une musique - les métaphores sont ici les expressions justes et nécessaires d'une poésie intime -, il n'y a rien (à dire) au-delà.

10. J.-M. Schaeffer, ouvr. cité, p. 185.

11. Schwob démarque ici le titre du recueil d'articles de P. Quillard, La Gloire du Verbe (1885-1890), Paris, Librairie de L'Art indépendant, 1890.

12. W. Iser, L'acte de lecture: théorie de l'effet esthétique, Bruxelles, Pierre Mardaga, coll. «Philosophie et langage », 1985.

13. J. de Palacio, Les perversions du merveilleux. Ma Mère l'Oye au tournant du siècle, Paris, Séguier, 1993.

14. M. Kundera, «Le jour où Panurge ne fera plus rire », dans Les Testaments trahis, Gallimard, coll. « Folio », 1993, p. 27.

15. Je laisse ici de côté l'étayage imaginaire qui constitue la mythologie propre à Kundera sur le roman. Ce n'est pas la proposition sur les œuvres de Rabelais comme pierres fondatrices de l'histoire du roman qu'il faut retenir ici - chez le même Kundera, ce sera tout aussi bien ailleurs Don Quichotte de Cervantès, au service d'une même mythologie édifiée par d'autres voies -, mais la prise en compte du processus de réappropriation en tant qu'il fait partie intégrante d'une histoire dans laquelle la perception du présent reconfigure continuellement le passé.

16. On consultera avec profit, quitte à le discuter, le répertoire voulu exhaustif établi dans la thèse d'Hermeline Pernoud, Féeries pour une autre fois : réécritures et renouvellement des paradigmes des contes de fées (1808-1920), Université Sorbonne Nouvelle - Paris 3, 2017, p. 693-749.

17. Sur ce point, voir B. Vibert, « Pour une poétique du recueil », dans Poète, même en prose, ouvr. cité, p. 323-380.

18. Voir plus haut note 6, en particulier les ouvrages de Chr. Leroy et N. Vincent-Munnia. Voir aussi N. Vincent-Munnia, S. Bernard-Griffiths et R. Pickering (dir.), Aux origines du poème en prose français : 1750-1850, Paris, H. Champion, coll. « Romantisme et Modernités », 2003.

19. Sur la notion, voir La verve, textes recueillis et présentés par B. Vibert, Recherches et Travaux, $\mathrm{n}^{\circ}$ 85, Grenoble, ELLUG, 2014. 


\section{AUTEUR}

\section{BERTRAND VIBERT}

Université Grenoble Alpes.

Bertrand Vibert est professeur de littérature française à l'Université Grenoble Alpes. Il a consacré une partie de ses travaux à la poétique historique des genres et à l'étude des recueils de contes poétiques en prose de la période symboliste. Il est l'auteur de Poète, même en prose (Paris, Presses universitaires de Vincennes, coll. «L'Imaginaire du Texte », 2010) et il a dirigé la publication en trois volumes de six recueils de contes symbolistes aux ELLUG (désormais UGA Éditions), respectivement publiés en 2009, 2011 et 2016, et consacrés à des recueils de Bernard Lazare, Marcel Schwob, Remy de Gourmont, Henri de Régnier, Georges Rodenbach et Camille Mauclair. 